\title{
Necessary and Sufficient Conditions for Complementary Stochastic Quadratic Operators of Finite-Dimensional Simplex
}

\author{
Rawad Abdulghafor, Sherzod Turaev, Akram Zeki, \\ Collage of Information and Communication Technology, International Islamic \\ University Malaysia, 53100,Kuala Lumpur, Malaysia \\ raaac2004@yahoo.com \\ sherzod@iium.edu.my,_akramzeki@iium.edu.my
}

\begin{abstract}
We define a complementary stochastic quadratic operator on finite-dimensional space as a new sub-class of quadratic stochastic operator. We give necessary and sufficient conditions for complementary stochastic quadratic operator.
\end{abstract}

Keywords: Quadratic stochastic operators, complementary stochastic quadratic operator, finite-dimensional space, Sub-stochastic matrix

\section{Introduction}

A quadratic stochastic operator is a general nonlinear model traced back to [1]. A lot of research have been devoted to investigations of various sub-classes of quadratic stochastic operator such as doubly stochastic quadratic operators, dissipative of quadratic stochastic operators, volterra quadratic stochastic operators and extreme doubly stochastic quadratic operators [2]-[7]. In recent years, this theory has become of a great interest in its multiple applications to the problems of population genetics [8]-[10] and control systems [11], [12]. During the past 80 years, nonlinear models have been focused in many researches due to their efficiency as well as their advantages over linear models [13], [14].

In effect, this motivates the study of a new nonlinear model in this paper. The central and main problem in nonlinear models of the family classes of quadratic stochastic operator is to study the limit behaviour their related trajectories. This is true as such nonlinear have complicated structure. This paper focuses on defining a nonlinear model with less complex structure.

\section{Preliminaries}

A quadratic stochastic operator is formed as follows:

$$
(\mathrm{Vx})_{\mathrm{k}}=\sum_{\mathrm{i}, \mathrm{j}=1}^{\mathrm{m}} \mathrm{p}_{\mathrm{ij}, \mathrm{k}} \mathrm{x}_{\mathrm{i}} x_{j},
$$

where

$$
\begin{aligned}
x \in S^{m-1}=\{x= & \left(x_{1}, x_{2}, \ldots, x_{m}\right) \in R^{m} \\
& : x_{i} \geq 0, \forall_{i} \\
& =\overline{1, m}, \sum_{i=1}^{m} x_{i} \\
& =1\} \quad(2)
\end{aligned}
$$

and the coefficients $p_{i j, k}$ satisfy the conditions 


$$
\begin{aligned}
p_{i j, k}=p_{j i, k} \geq 0, & \sum_{\substack{k=1 \\
=1}}^{m} p_{i j, k} \\
&
\end{aligned}
$$

A quadratic stochastic operator is defined on a free population space. In meaning, suppose that the free space of population involves a set of $m$ elements. This set is defined on a simplex as in Equation 2, and it is termed as an ( $m-$ 1)-dimensional simplex.

A quadratic stochastic operator assigned the same simplex, $V: S^{m-1} \rightarrow$ $S^{m-1}$, is formed as in Equation 1.

The maps among the elements $x_{i}$ are considered as a distributed stochastic matrix given by

$$
\begin{gathered}
p_{i j, k}= \\
\left(p_{i j, 1}, p_{i j, 2}, \ldots, p_{i j, k}\right)
\end{gathered}
$$

where $p_{i j, k}$ is considered under the conditions of Equation 3.

In this paper, we define a new model of complementary stochasticity quadratic operators from the general model of quadratic stochastic operator under some derived some conditions included in $p_{i j, k}$. The concept of the complementary stochasticity quadratic operators is explored in the next section.

\section{Complementary Stochasticity Quadratic Operators}

As known that $p_{i j, k}$ is a stochastic matrix of the distribution matrices $\left(p_{i j, 1}, p_{i j, 2}, \ldots, p_{i j, k}\right)$ in the operator $V(x): S^{m-1} \rightarrow S^{m-1}$ as given in Equation 1 .
Definition: An operator $V(x)$ is called complementary stochasticity quadratic if has a complementary stochastic matrix $P$, then $V(x)=P x$, where $P$ is a matrix $(\mathrm{m} \times m) . P=\left[p_{i j, k}\right]$ is said to be a complementary stochastic matrix if

i) for all $1 \leq i \neq \leq m, p_{i j, k}=p_{j i, k}=0$ or $p_{j i, k} \neq 0$ and $p_{j i, k}=1-p_{i j, k}$.

ii) For all1 $\leq i \leq m, p_{i i, k}=0$ or $p_{i i, k}=\frac{1}{2}$.

iii) For all distributed matrices $P_{i j, k}=$ $\left(p_{i j, 1}, p_{i j, 2}, \ldots, p_{i j, k}\right), \sum_{i j=1}^{m} p_{i j, k}=$ $m, \sum_{k=1}^{m} p_{i j, k}=1$.

Therefore, the new notations for complementary stochasticity quadratic operators for matrices $\left(p_{i j, 1}, p_{i j, 2}, \ldots, p_{i j, k}\right)$ are

$$
U_{\text {low }}=\left\{\begin{array}{c}
p_{i i, k}=\frac{1}{2} \vee 0, p_{j i, k}=1-p_{i j, k} \text { or } 0, \\
\sum_{i j=1}^{m} p_{i j, k}=m, \sum_{k=1}^{m} p_{i j, k}=1
\end{array}\right\}
$$

where $p_{i i, k}$ are the diagonal elements limited to either 0 or $\frac{1}{2}$, each symmetric element is stochastic $p_{i j, k}+p_{j i, k}=1$ with respect that the elements' sum of each distributed matrix $p_{i j, k}$ begin equal to $m$ and the summation of all distributed matrices $\left(p_{i j, 1}, p_{i j, 2}, \ldots, p_{i j, k}\right)$ is a matrix that has all of its elements equal to 1 .

The key idea in complementary stochasticity quadratic operators is to make the coefficient of the elements equal to 1 or0. The distributed matrices $p_{i j, k}=$ $\left(p_{i j, 1}, p_{i j, 2}, \ldots, p_{i j, k}\right)$ are structured as

$\left(\begin{array}{cccc}a_{11,1} & a_{12,1} & \ldots & a_{1 m, 1} \\ 1-a_{12,1} & a_{22,1} & \ldots & a_{2 m, 1} \\ \vdots & \vdots & \ddots & \vdots \\ 1-a_{1 m, 1} & 1-a_{2 m, 1} & \ldots & a_{m m, 1}\end{array}\right)+$ 
R. Abdulghafor et al. Necessary and Sufficient Conditions for Complementary Stochastic Quadratic Operators of

$\left(\begin{array}{cccc}a_{11,2} & a_{12,2} & \ldots & a_{1 m, 2} \\ 1-a_{12,2} & a_{22,2} & \ldots & a_{2 m, 2} \\ \vdots & \vdots & \ddots & \vdots \\ 1-a_{1 m, 2} & 1-a_{2 m, 2} & \ldots & a_{m m, 2}\end{array}\right)+$

$\left(\begin{array}{cccc}\cdots & \cdots & \cdots & \cdots \\ \cdots & \cdots & \cdots & \cdots \\ \vdots & \vdots & \ddots & \vdots \\ \cdots & \cdots & \cdots & \ldots\end{array}\right)+$

$\left(\begin{array}{cccc}a_{11, m} & a_{12, m} & \ldots & a_{1 m, m} \\ 1-a_{12, m} & a_{22, m} & \ldots & a_{2 m, m} \\ \vdots & \vdots & \ddots & \vdots \\ 1-a_{1 m, m} & 1-a_{2 m, m} & \ldots & a_{m m, m}\end{array}\right)=$

$\left(\begin{array}{cccc}1 & 1 & \cdots & 1 \\ 1 & 1 & \cdots & 1 \\ \vdots & \vdots & \ddots & \vdots \\ 1 & 1 & \cdots & 1\end{array}\right)$

referring to the evaluation operator in Equation 1, the analytical procedure of this method is as follows:

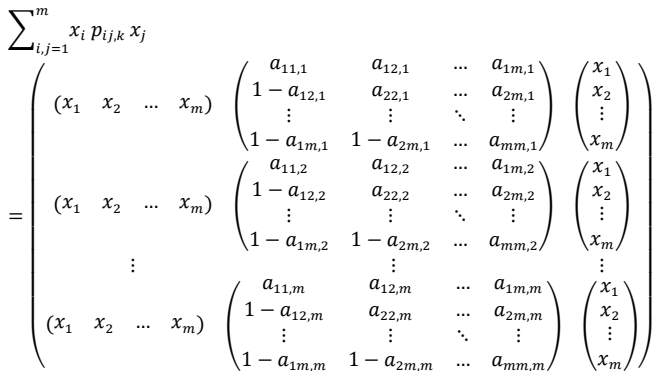

where $a_{i j}$ is the coefficient among the two points $x_{i}$ and $x_{j}$ and $\left(x_{1}, x_{2}, \ldots, x_{m}\right)$ are vector of points.

Then, the evaluation of the nonlinear operator $V(x)$ is calculated as follows:

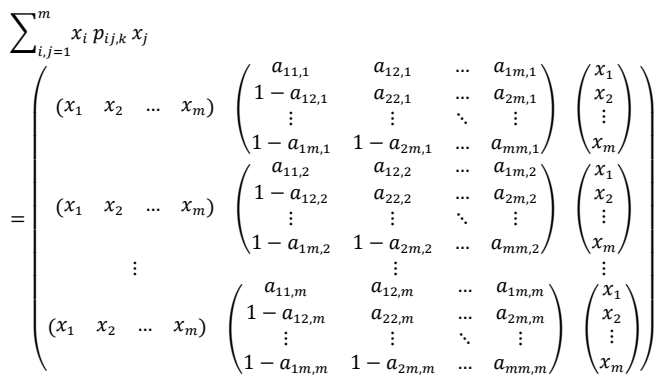

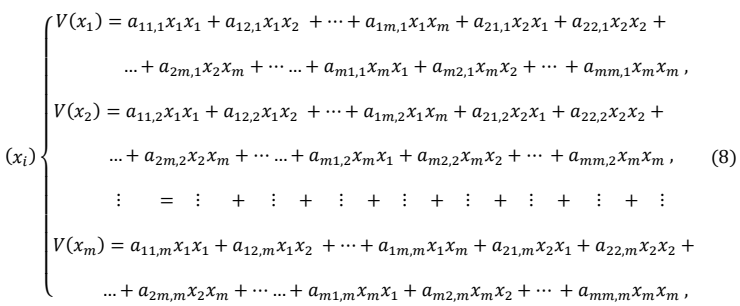

\section{Some Examples Of Complementary Stochasticity Quadratic Operators}

1. Example 1, operator $V_{1}(x)$ with $m=3$ :

In the case of distributed matrices $p_{i j, k}$, there are three as follows

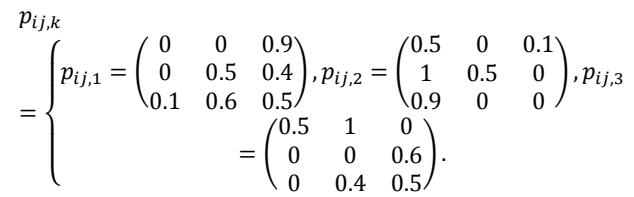

Using Equation (7) then we get

$$
V_{1}(x)\left\{\begin{array}{l}
X_{1}=x_{1} x_{3}+x_{2} x_{3}+\frac{x_{2}^{2}}{2}+\frac{x_{3}^{2}}{2} \\
X_{2}=x_{1} x_{2}+x_{1} x_{3}+\frac{x_{1}^{2}}{2}+\frac{x_{2}^{2}}{2} \\
X_{3}=x_{1} x_{2}+x_{2} x_{3}+\frac{x_{1}^{2}}{2}+\frac{x_{3}^{2}}{2}
\end{array}\right.
$$

2. Example 2, operator $V_{2}(x)$ with $m=3$ : In the case of distributed matrices $p_{i j, k}$, then there are three and given by

$$
=\left\{\begin{array}{c}
p_{i j, k}=\left(\begin{array}{ccc}
0 & 0.5 & 0.5 \\
0.5 & 0.5 & 0 \\
0.5 & 0 & 0.5
\end{array}\right), p_{i j, 2}=\left(\begin{array}{ccc}
0.5 & 0.5 & 0 \\
0.5 & 0 & 0.5 \\
0 & 0.5 & 0.5
\end{array}\right), \\
p_{i j, 3}=\left(\begin{array}{ccc}
0.5 & 0 & 0.5 \\
0 & 0.5 & 0.5 \\
0.5 & 0.5 & 0
\end{array}\right) .
\end{array}\right.
$$

Similarly, sing Equation (7) then we get 


$$
V_{2}(x)\left\{\begin{array}{l}
X_{1}=x_{1} x_{2}+x_{1} x_{3}+\frac{x_{2}{ }^{2}}{2}+\frac{x_{3}{ }^{2}}{2} \\
X_{2}=x_{1} x_{2}+x_{2} x_{3}+\frac{x_{1}{ }^{2}}{2}+\frac{x_{3}{ }^{2}}{2} \\
X_{3}=x_{1} x_{3}+x_{2} x_{3}+\frac{x_{1}{ }^{2}}{2}+\frac{x_{2}{ }^{2}}{2}
\end{array}\right.
$$

3. Example 3, operator $V_{3}(x)$ with $m=4$ : In the case of distributed matrices $p_{i j, k}$, there are four

$$
=\left\{\begin{array}{c}
p_{i j, 1}=\left(\begin{array}{cccc}
0.5 & 0 & 0 & 0.2 \\
0 & 0.5 & 0 & 0 \\
0 & 0 & 0.5 & 0.6 \\
0.8 & 0 & 0.4 & 0.5
\end{array}\right), p_{i j, 2}=\left(\begin{array}{cccc}
0.5 & 1 & 0.7 & 0 \\
0 & 0 & 0 & 0.6 \\
0.3 & 0 & 0.5 & 0 \\
0 & 0.4 & 0 & 0
\end{array}\right), \\
p_{i j, 3}=\left(\begin{array}{cccc}
0 . & 0 & 0.3 & 0 \\
0 & 0 & 0 & 0.4 \\
0.7 & 1 & 0 & 0.4 \\
0 & 0.6 & 0.6 & 0
\end{array}\right), p_{i j, 4}=\left(\begin{array}{cccc}
0 & 0 & 0 & 0.8 \\
1 & 0.5 & 1 & 0 \\
0 & 0 & 0 & 0 \\
0.2 & 0 & 0 & 0.5
\end{array}\right) .
\end{array}\right.
$$

Using Equation (7) again, we get

$$
V_{3}(x)\left\{\begin{array}{l}
X_{1}=x_{1} x_{4}+x_{3} x_{4}+\frac{x_{1}^{2}}{2}+\frac{x_{2}^{2}}{2}+\frac{x_{3}^{2}}{2}+\frac{x_{4}^{2}}{2} \\
X_{2}=x_{1} x_{2}+x_{1} x_{3}+x_{2} x_{4}+\frac{x_{1}^{2}}{2}+\frac{x_{3}^{2}}{2} \\
X_{3}=x_{1} x_{3}+x_{2} x_{3}+x_{2} x_{4}+x_{3} x_{4} \\
X_{4}=x_{1} x_{2}+x_{1} x_{4}+x_{2} x_{3}+\frac{x_{2}^{2}}{2}+\frac{x_{4}^{2}}{2}
\end{array}\right.
$$

4. Example 4, operator $V_{4}(x)$ with $m=4$ : In the case of distributed matrices $p_{i j, k}$, there are four

$$
\begin{aligned}
& p_{i j, k} \\
& p_{i j, 1}=\left(\begin{array}{cccc}
0 & 0.6 & 0.4 & 0 \\
0.4 & 0 & 0 & 0.6 \\
0.6 & 0 & 0 & 0.4 \\
0 & 0.4 & 0.6 & 0
\end{array}\right), p_{i j, 2}=\left(\begin{array}{cccc}
0 & 0.4 & 0.6 & 0 \\
0.6 & 0 & 0 & 0.4 \\
0.4 & 0 & 0 & 0.6 \\
0 & 0.6 & 0.4 & 0
\end{array}\right), \\
& p_{i j, 3}=\left(\begin{array}{cccc}
0.5 & 0 & 0 & 0.5 \\
0 & 0.5 & 0.5 & 0 \\
0 & 0.5 & 0.5 & 0 \\
0.5 & 0 & 0 & 0.5
\end{array}\right), p_{i j, 4}=\left(\begin{array}{cccc}
0.5 & 0 & 0 & 0.5 \\
0 & 0.5 & 0.5 & 0 \\
0 & 0.5 & 0.5 & 0 \\
0.5 & 0 & 0 & 0.5
\end{array}\right) .
\end{aligned}
$$

Using Equation (7) we get

$$
\begin{aligned}
& \left\{\begin{array}{l}
X_{1}=x_{1} x_{2}+x_{1} x_{3}+x_{2} x_{4}+x_{3} x_{4} \\
X_{2}=x_{1} x_{2}+x_{1} x_{3}+x_{2} x_{4}+x_{3} x_{4}
\end{array}\right. \\
& X_{3}=\begin{array}{l}
x_{1} x_{4}+x_{2} x_{3}+\frac{x_{1}{ }^{2}}{2}+ \\
\frac{x_{2}{ }^{2}}{2}+\frac{x_{3}{ }^{2}}{2}+\frac{x_{4}{ }^{2}}{2}
\end{array} \\
& X_{4}=\begin{array}{l}
x_{1} x_{4}+x_{2} x_{3}+\frac{x_{1}{ }^{2}}{2} \\
+\frac{x_{2}{ }^{2}}{2}+\frac{x_{3}{ }^{2}}{2}+\frac{x_{4}{ }^{2}}{2}
\end{array}
\end{aligned}
$$

5. Example 5, operator $V_{5}(x)$ with $m=5$ :

In the case of distributed matrices $p_{i j, k}$, then there are five as

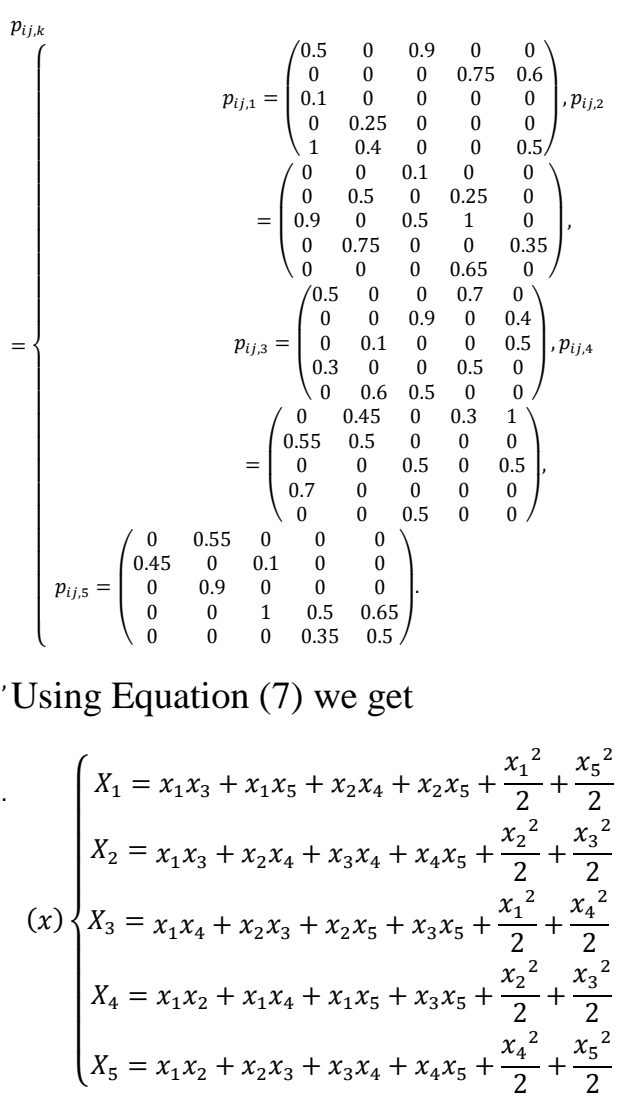

6. Example 6, operator $V_{6}(x)$ with $m=5$ : 
In the case of distributed matrices $p_{i j, k}$, then there are four as follows

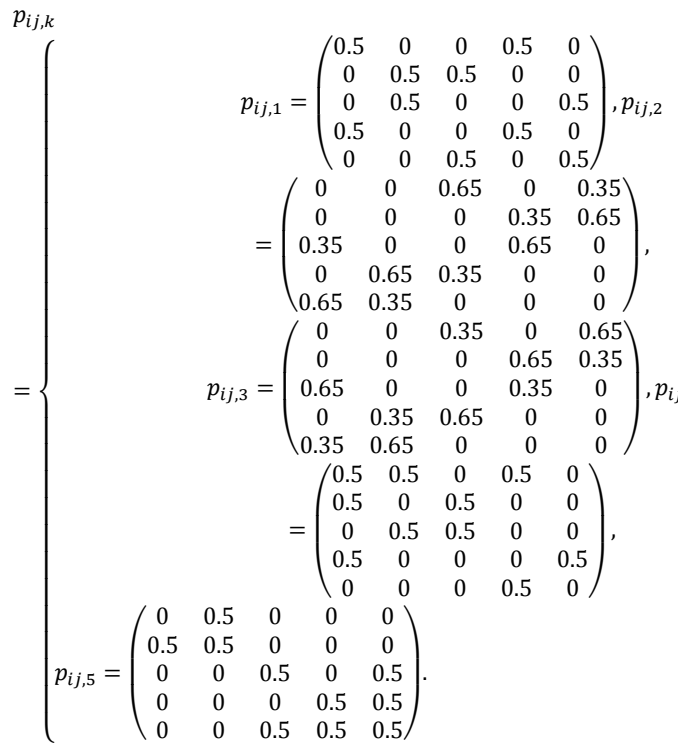

Using Equation (7) we get

$$
(x)\left\{\begin{array}{c}
X_{1}=x_{1} x_{4}+x_{2} x_{3}+x_{3} x_{5} \\
+\frac{x_{1}{ }^{2}}{2}+\frac{x_{2}{ }^{2}}{2}+\frac{x_{4}{ }^{2}}{2}+\frac{x_{5}{ }^{2}}{2} \\
X_{2}=x_{1} x_{3}+x_{1} x_{5}+x_{2} x_{4}+x_{2} x_{5}+x_{3} x_{4} \\
X_{3}=x_{1} x_{3}+x_{1} x_{5}+x_{2} x_{4}+x_{2} x_{5}+x_{3} x_{4} \\
X_{4}=x_{1} x_{2}+x_{1} x_{4}+x_{2} x_{3}+x_{4} x_{5} \\
+\frac{x_{1}{ }^{2}}{2}+\frac{x_{3}{ }^{2}}{2} \\
X_{5}=x_{1} x_{2}+x_{3} x_{5}+x_{4} x_{5}+\frac{x_{2}{ }^{2}}{2} \\
+\frac{x_{3}{ }^{2}}{2}+\frac{x_{4}{ }^{2}}{2}+\frac{x_{5}{ }^{2}}{2}
\end{array}\right.
$$

\section{Conclusion}

In this paper, the new nonlinear model of complementary stochasticity quadratic operators has been established. The sufficient conditions and notations have in turn been defined to investigate the complementary stochasticity quadratic operators. In fact, the less complex structure in this model has been considered.

\section{Acknowledgment}

We would like to thank the Kulliyyah of Information and Communication Technology, as well as the Research Management Center of the International Islamic University Malaysia, for the support. This work was funded by the International Islamic University of Malaysia through IIUM Endowment B research grant EDWB13-053-0938.

\section{References}

[1] S. Bernstein, "Solution of a mathematical problem connected with the theory of heredity," Ann. Math. Stat., vol. 13, no. 1, pp. 53-61, 1942.

[2] R. Ganikhodzhaev and F. Shahidi, "Doubly stochastic quadratic operators and Birkhoff's problem," Linear Algebra Appl., vol. 432, no. 1, pp. 24-35, 2010.

[3] F. Shahidi, "On dissipative quadratic stochastic operators," Appl. Math. Inf. Sci., vol. 2, pp. 211-223, 2008.

[4] R. Abdulghafor, S. Turaev, A. Abubakar, and A. Zeki, "The Extreme Doubly Stochastic Quadratic Operators on Two Dimensional Simplex," 2015 4th Int. Conf. Adv. Comput. Sci. Appl. Technol., pp. 192-197, 2015.

[5] F. Shahidi, R. Ganikhodzhaev, and R. Abdulghafor, "The Dynamics of Some Extreme Doubly Stochastic Quadratic Operators," MiddleEast J. Sci. Res. (Mathematical Appl. Eng., vol. 13, pp. 59-63, 2013.

[6] F. Mukhamedov and A. F. Embong, "On b-bistochastic quadratic stochastic operators," J. Inequalities Appl., vol. 2015, no. 1, p. 226, 2015.

[7] U. A. Rozikov and U. U. Zhamilov, "Volterra quadratic stochastic operators of a twosex population," Ukr. Math. J., vol. 63, no. 7, pp. 1136-1153, 2011. 
[8] R. N. Ganikhodzhaev and U. A. Rozikov, "Quadratic Stochastic Operators: Results and Open Problems," arXiv Prepr. arXiv0902.4207, 2009.

[9] R. Abdulghafor, F. Shahidi, A. Zeki, and S. Turaev, "Dynamics of doubly stochastic quadratic operators on a finite-dimensional simplex," Open Math. Gruyter Open., vol. 14, no. 1, pp. 509-519, 2016.

[10] S. Abdulghafor, Rawad and Shahidi, Farruh and Zeki, Akram and Turaev, "Dynamics Classications of Extreme Doubly Stochastic Quadratic Operators on 2D Simplex," Adv. Comput. Commun. Eng. Technol. Springer, pp. 323--335, 2016.

[11] S. Abdulghafor, Rawad and Shahidi, Farruh and Zeki, Akram and Turaev, "The Convergence Consensus of Multi-agent Systems Controlled via Doubly Stochastic Quadratic Operators," Agents, Multi-Agent Syst. Robot. (ISAMSR), 2015 Int. Symp. on, IEEE, pp. 59--64, 2015.

[12] R. Abdulghafor, S. Turaev, and $M$. Izzuddin, "Nonlinear Consensus for Multi-Agent Systems using Positive Intractions of Doubly Stochastic Quadratic Operators," Int. J. Perceptive Cogn. Comput., vol. 2, no. 1, pp. 19-22, 2016.

[13] G. Cui, S. Xu, F. L. Lewis, B. Zhang, and Q. Ma, "Distributed consensus tracking for nonlinear multi-agent systems with input saturation: a command filtered backstepping approach," IET Control Theory Appl., 2016.

[14] L. Yu-Mei and G. Xin-Ping, "Nonlinear consensus protocols for multi-agent systems based on centre manifold reduction," Chinese Phys. B, vol. 18, no. 8, p. 3355, 2009. 